Nouvelles perspectives en sciences sociales

Revue internationale de systémique complexe et d'études relationnelles

\title{
Patrimoine toponymique des minorités culturelles, lieu de complexités : le cas de la Fransaskoisie
}

\section{Carol Jean Léonard}

Volume 6, numéro 1, décembre 2010

Sur le thème de la minorité linguistique

URI : https://id.erudit.org/iderudit/1000484ar

DOI : https://doi.org/10.7202/1000484ar

Aller au sommaire du numéro

\section{Éditeur(s)}

Prise de parole

ISSN

1712-8307 (imprimé)

1918-7475 (numérique)

Découvrir la revue

Citer cet article

Léonard, C. J. (2010). Patrimoine toponymique des minorités culturelles, lieu de complexités : le cas de la Fransaskoisie. Nouvelles perspectives en sciences sociales, 6(1), 99-124. https://doi.org/10.7202/1000484ar

\section{Résumé de l'article}

Le présent article examine successivement deux grandes questions que pose la toponymie en tant que référentiel identitaire. Dans un premier temps, et après avoir évoqué l'évolution récente de la toponymie qui en a établi les fondements en tant que science transdisciplinaire autonome, nous identifions les apories identificatoires auxquelles se heurte toute tentative d'inventaire exhaustif d'une toponymie s'appuyant sur la langue et sur la culture en situation multilingue et de partage d'un territoire. Nous dressons une typologie des entraves qui gênent l'identification des noms géographiques en relation avec une culture cible. Enfin, nous illustrons comment toute tentative en vue de dresser la nomenclature exhaustive d'un patrimoine toponymique culturel identitaire conduit à dénouer l'écheveau d'un capital hétérogène marqué du sceau de l'interculturalité. Aux fins d'illustration, nous prenons à témoin les obstacles rencontrés lors de l'inventaire des 2500 toponymes d'origine et d'influence françaises identifiés en Saskatchewan, province sise au centre du Canada. Ces toponymes serviront également d'exemples. 


\title{
Patrimoine toponymique des minorités culturelles, lieu de complexités : le cas de la Fransaskoisie
}

\author{
Carol Jean Léonard \\ Université de l'Alberta, Edmonton
}

\begin{abstract}
Tncontournables, puisqu'ils répondent à un besoin fondamen$\mathrm{tal}^{1}$, les toponymes constituent l'un des éléments les plus essentiels et les plus pérennes du paysage linguistique ambiant. Indispensables à l'orientation et à la localisation, ils peuplent les cartes, abondent dans la signalisation routière, émaillent les conversations ${ }^{2}$. Leur fréquentation constante les range au nombre des éléments qui s'intègrent et se fondent le mieux au quotidien. Statiques et en apparence immuables, ils en viennent à n'apparaître plus que sous les traits du familier et de l'anodin. La curiosité ne porte souvent sur eux qu'au moment de dénouer le sens de ceux d'entre eux qui apparaissent obscurs, incongrus.
\end{abstract}

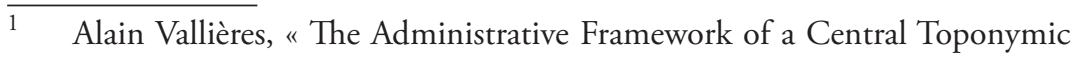
Agency ", Canadian Documents for the United Nations Toponymy Courses, Ottawa, 1992, p. 46.

2 Le toponymiste Henri Dorion rappelle qu'une seule édition du New York Times peut renfermer jusqu’à 2000 noms de lieux. Voir Henri Dorion, «Les noms de lieux, repères indispensables dans l'espace et dans le temps ", Discours de conférencier prononcé à l'occasion du Géocongrès international, Québec, http://www.quebec2007.ca/pdf/salle2000aetb/dinermercredi/h_dorion. pdf, (consulté le 20 juillet 2009). 
En situation de contact des cultures et des langues, l'attention peut se focaliser sur l'ensemble d'un corpus pour en dégager ce qui participe à la singularisation d'un groupe, pour discerner ce qui, dans le marquage effectif et symbolique de l'espace, permet de mesurer l'étendue et la durée de l'occupation de ce groupe sur le territoire en partage. On cherchera alors à établir sa continuité historique ou à découvrir ce qui, dans la toponymie ambiante, le rattache encore à son passé et qui permet d'apprécier l'influence qu'il a pu avoir sur la manière de nommer le pays. Les régions de contact et d'interpénétration culturelle prédisposent à ce type d'interrogation. Les noms géographiques y revêtent un attribut complémentaire : ils constituent un référentiel appropriable, source d'identisation ${ }^{3}$ et d'identification.

Des études récentes le démontrent à l'évidence ${ }^{4}$; les rapports que les chercheurs, les gestionnaires publics et même les usagers entretiennent avec la toponymie sont en pleine mutation. Si l'on ne perçoit encore que très imparfaitement les enjeux que ces nouvelles perspectives dessinent, cela tient en partie au fait d'une attention des toponymistes trop longtemps restée fixée sur une toponymie objet d'inventaire, de gestion et d'étymologie. On a ainsi longtemps fait abstraction de l'influence qu'exerce cet important référentiel identitaire lors même que les noms de lieux occupent une place importante au cœur du paysage linguistique ambiant $^{5}$. Nul ne peut se soustraire à l'usage de toponymes. Et pourtant, leur influence s'exerce sans qu'elle ait pour cela à s'exprimer dans le champ de la conscience. Le nom de lieu

3 Pierre Tap définit l'identisation comme étant « le processus par lequel l'acteur social s'intègre à un ensemble plus vaste et dans lequel il tend à se fondre ». Pierre Tap (dir.), Identité collective et changements sociaux, Toulouse, Privat, 1979, p. 11.

4 Carol Jean Léonard, «Le patrimoine toponymique minoritaire d'origine et d'influence française à l'école : une voie de construction identitaire à emprunter ", Éducation francophone en milieu minoritaire, vol. 31, $\mathrm{n}^{\circ} 1$, 2008, p. 15-32.

5 Carol Jean Léonard, «Une toponymie voilée : problématique des noms de lieux particulière à une minorité canadienne, la Fransaskoisie ", thèse de doctorat, Québec, Université Laval, 2006. 
s'intègre si parfaitement aux usages que leur fonction de localisation prend le pas sur leur fonction symbolique. Elle prévaut sur elle, sans la dominer. C'est dans la réification de la charge symbolique du nom que ce dernier puise sa puissance, qu'il véhicule et légitime une conception du monde, de l'histoire et des rapports intergroupes. En situation de contact des cultures et des langues, la toponymie révèle souvent l'état de vitalité ethnolinguistique des groupes en présence autant qu'elle reflète la nature de leurs rapports sur l'échiquier social et politique ${ }^{6}$. Plus encore, elle concourt à en définir les termes et leur donne corps sensible. Inévitable parce qu'indispensable à la localisation et à l'orientation, le nom s'impose à l'usage qui, en retour, le valide tout comme il légitime la langue, l'histoire et la domination qu'il symbolise, que celle-ci soit ethnoculturelle ou glottopolitique.

C'est précisément cet horizon qui a inspiré des chercheurs à vouloir opérer la jonction entre les travaux théoriques sur le pouvoir symbolique du langage et ceux plus prosaïques d'enquête sur le terrain ${ }^{7}$. Ils contribuent ainsi à l'affranchissement de la toponymie autrefois si souvent réduite à des fonctions ancillaires au bénéfice premier de la linguistique. Le recentrement de la toponymie au carrefour des disciplines que sont l'histoire, la géographie et la linguistique a ainsi permis d'élargir le spectre des voies complémentaires et transdisciplinaires par lequel le nom de lieu, son action et son pouvoir peuvent être appréhendés. L'on assiste aujourd'hui à l'émergence d'une pragmatique de la toponymie. Au cours des récentes décennies, les études traitant de la toponymie selon des perspectives politiques, sociolinguistiques et même géopolitiques se sont accumulées.

$6 \quad$ Richard Y. Bourhis, Howard Giles et Doreen Rosenthal, " Notes on the Construction of A "Subjective Vitality Questionnaire” For Ethnolinguistic Groups ", Journal of Multilingual and Multicultural Development, vol. 2, 1981, p. 145-155.

7 Jani Vuolteenaho et Lawrence D. Berg, Towards Critical Toponymies Chapter 1, http://www.ashgatepublishing.com/pdf/SamplePages/Critical_ Toponymies_Ch1.pdf, (consulté le 15 juillet 2009).

8 Naftali Kadmon, "Toponymy and Geopolitics: The Political Use - and Misuse - of Geographical Names ", The Cartographic Journal, vol. 41, $\mathrm{n}^{\circ} 2$, 2004, p. 85-87.Voir également les articles suivants : Wilhelm Fritz Hermann 
Euvrer au dénombrement des noms de lieux qui ressortissent à une langue et à une culture, les rassembler en des corpus cohérents, irréfragables et englobants pose des défis conceptuels et méthodologiques considérables. On ne peut procéder comme on le fait pour les toponymies territoriales dont on peut aisément délimiter le champ des inventaires en prenant appui sur les frontières juridiques. La recension des noms de lieux sis à l'intérieur de l'aire qu'ils délimitent constitue l'essentiel de la tâche. Il en va tout autrement des toponymies culturelles et identitaires qui, elles, reposent sur les notions fluides de langue et de culture pour lesquelles il ne peut y avoir ni de frontières clairement établies, ni de définition normative ou prescriptive a posteriori. Définir et circonscrire posent certes des difficultés. En revanche, pareille tâche ouvre des perspectives sur l'état du partage des territoires comme sur celui de l'hétérogénéité des toponymies qui s'y rencontrent ${ }^{9}$. C'est du reste le lot de la vaste majorité des régions du globe d'avoir été traversée et occupée par des groupes de locuteurs de langues étrangères ou apparentées les unes aux autres. Les toponymies issues de ces passages et de ces occupations peuvent présenter des aspects si divers, d'un métissage si varié, pourrait-on dire, qu'on ne peut en rendre aisément toute la complexité. Les zones qu'elles occupent et chevauchent, les rapports qu'elles entretiennent s'accordent aux cadres historiques, géographiques, politiques et juridiques qui s'y observent. Aux entraves se rapportant à l'identification des toponymes eux-mêmes s'ajoutent celles ressortissant au tri des noms. L'on comprend dès lors la mise à contribution de l'ensemble des sciences aptes à "faire parler " les toponymes pour parvenir à dénouer pareils écheveaux.

Nicolaisen, "Placenames and Politics », Names, vol. 38, n 3, 1990, p. 193207; Henri Dorion, "La toponymie, complice involontaire de la politique ", dans Proceedings of the XIXth International Congress of Onomastic Sciences, Aberdeen, 4 au 11 août 1996, Department of English, University of Aberdeen, 1998, p. 5-19.

9 Henri Dorion et Christian Morrissonneau (dir.), Les noms de lieux et le contact des langues. Place Names and Language Contact, Québec, Presses de l'Université Laval, 1972, p. 9-41. 
Le paysage toponymique de la Saskatchewan et l'état de la toponymie fransaskoise ${ }^{10}$ fournissent les éléments d'un cadre approprié à l'analyse des difficultés propres à la recension d'un corpus dont se réclame un groupe linguistique appartenant à une minorité de langue officielle au Canada. Comme celles de l'ensemble des provinces canadiennes à l'exception du Québec, la toponymie de la Saskatchewan est fortement marquée par la langue de la majorité anglophone. Les termes génériques des noms de lieux y sont, à quelques exceptions près, tous en anglais. Les spécifiques en revanche offrent une plus grande diversité. Bien que ceux en langue anglaise soient en nette majorité, ils en côtoient d'autres en langues crise, déné, sauteuse, lakota, allemande, ukrainienne et française. Cette composition reflète l'état du groupe francophone en situation de partage inégal des langues. Cette minorité n’y représentait en 2001 qu'environ 1,9 \% d'une population entière dont le nombre $\mathrm{d}^{\prime}$ habitants était alors légèrement inférieur au million ${ }^{11}$.

L'étude des toponymes français ${ }^{12}$ a permis la recension de près de 2500 noms de lieux historiques et contemporains d'origine et d'influence françaises dans cette province où le nombre de toponymes officiels n'atteint pas les 12500 (figure 1). Ces chiffres traduisent l'influence historique qu'a pu connaître la langue française dans cette région bien avant même qu'elle ait acquis le statut de province en $1905^{13}$. La mise en rapport de ces chiffres permet d'entrevoir l'influence qu'a pu connaître la langue française dans cette région tout au long de son histoire.

$\overline{10}$ Depuis le début de leur présence sur le territoire saskatchewannais, les francophones furent connus sous diverses appellations : Canadiens, puis Canadiens-français, puis Franco-canadiens de la Saskatchewan. Depuis les années quatre-vingts, ils se désignent plus volontiers sous le vocable de Fransaskois. Nous employons donc l'adjectif fransaskois pour désigner la toponymie d'origine et d'influence françaises, présente et passée, sur le sol saskatchewannais.

11 Statistique Canada. Division des opérations du recensement 2002.

12 Carol Jean Léonard, "Une toponymie voilée... " op. cit.

13 Nombreux sont les toponymes sortis de l'usage. Seul le tiers des toponymes répertoriés (812) a été admis dans la nomenclature officielle. 
Figure 1

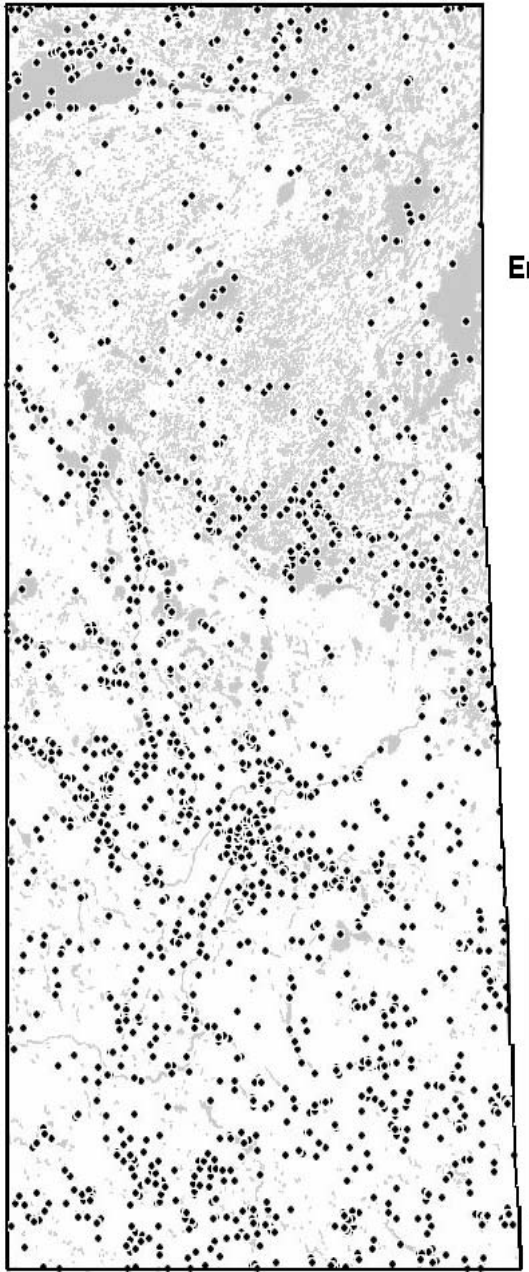

\section{SASKATCHEWAN}

De 1699 à 2000

Ensemble des lieux désignés par des toponymes d'origine et d'influence françaises

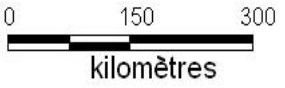

Sur un total de 2480 toponymes, la période d'apparition a pu être identifíée pour 1952 d'entre eux, soit près de pour exposer les données propres à chacun des segments de notre frise historique. Sur ce total de 1952 toponymes 867 d'entre eux, soit $44,4 \%$ \% se signalent au cours de la colonisation. L'efferyescence dénominative qui a marqué cette période s'et donc aussi reflétée dans la toponymie associée au fait frangais. Cette concordance s'observe également au cours de la période plus calme qui succède tout comme elle accompagne celle de Bereskin, marquée par un regain d'intérêt pour la chose toponymique. Les chiffres le confirment, l'époque contemporaine se montre peu propice à l'apparition de toponymes franģais.

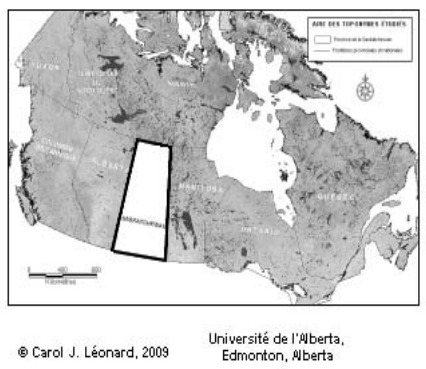

Tout au long des paragraphes qui suivent est présenté l'ensemble des entraves relevées lors de la saisie du corpus toponymique minoritaire fransaskois. Nous exposons celles qui relèvent des contraintes que connaît l'observateur du paysage linguistique, puis celles ressortissant à l'univers des mots eux-mêmes et à la dynamique évolutive des langues. 


\section{Critères d'inclusion}

Lidentification d'un patrimoine sur la base d'une appartenance à une langue et à une culture pose, nous venons de le souligner, des difficultés quant à la définition même de ce qu'est un toponyme français en pareil contexte. En contexte extra-hexagonal, identifier des toponymes "français ", c'est avant tout référer à une toponymie " en relation " pour laquelle il est nécessaire d'établir les liens qui valident l'épithète "française " et, par extension, "fransaskoise ". Il est question ici de liens avec la langue française bien entendu, de liens avec ses locuteurs, avec la France et son histoire et ses spécificités culturelles, mais aussi de liens avec ce qui constitue le prolongement historique, géographique et linguistique de la France en terre d'Amérique. Aussi, est-il est nécessaire ici d'exposer les critères définitoires retenus au cours de l'enquête sur la toponymie fransaskoise.

Ainsi, furent considérés d'origine et d'influence françaises :

- tout toponyme composé d'un ou de plusieurs mots appartenant au réservoir lexical de la langue française, qu’on les retrouve ou non parmi les termes génériques français empruntés par l'anglais nord-américain (prairie, coulée, butte, portage, rapides, etc.);

- tout toponyme qui, d'une manière incontestable, sans être exclusive, porte dans la graphie de son spécifique la marque du français;

- tout anthropotoponyme qui, d'une manière ou d'une autre, peut être associé à la langue française sans égard aux origines géographiques ni même aux attributs ou convictions linguistiques de celui qui l'a porté;

- tout toponyme commémoratif ou d'appartenance rappelant le souvenir d'une personne ayant eu le français pour langue maternelle ou d'adoption;

- tout toponyme constituant une transplantation d'un nom de lieu de France ou d'une région ailleurs dans le monde où ce toponyme est couramment associé à la langue française $e^{14}$.

14 Carol Jean Léonard, «Une toponymie voilée... », op. cit., p. 20-21. 


\section{Entraves}

Ces critères étant précisés, nous proposons ici une typologie des obstacles à l'identification des noms de lieux dans le contexte précédemment décrit et qui se sont dressés devant nous au cours de l'étude. Elle rend compte d'obstacles dont certains, incontournables, court-circuitent l'identification des toponymes et compromettent irrémédiablement leur interprétation et la reconstitution de leur genèse. D'autres, moins insurmontables, requièrent néanmoins pour être contournés une attention aux indices rendant possible leur reconnaissance en ayant recours à ce que l'arsenal des connaissances et des outils que les sciences opportunes au décodage peut offrir.

Ces entraves à l'appropriation sont de deux ordres. Il y a, d'une part, celles qui préviennent l'accès aux toponymes et, d'autre part, celles qui en gênent l'interprétation.

2.1. Entraves liées à l'accessibilité

Si le nom se présente comme un élément du langage, il ne peut être dissocié de l'espace qu'il désigne et de la période couverte par son usage. L'espace et le temps donnent corps au toponyme. Ils en conditionnent la saisie. Sur un territoire dont les premiers signes d'une occupation française intermittente remontent à près de 250 ans et dont la superficie $\left(651036 \mathrm{~km}^{2}\right)$ est comparable à celle de la France, l'accès aux toponymes passés ou en usage, puis leur identification ne se fait pas sans peine, particulièrement si cette enquête vise l'établissement d'un corpus aussi exhaustif que possible.

Entraves cognitivo-culturelles - On ne reconnaît que ce que l'on connaît. Voilà une difficulté toute simple, mais qui pèse de tout son poids. Plus vaste est la culture d'un individu, plus grande est sa capacité à interpréter les toponymes qui peuplent les cartes et s'affichent sur son chemin. En cela, la culture personnelle représente la première des contraintes à l'identification. La somme d'informations emmagasinées par chacun au cours de son existence connait une variabilité infinie d'un individu à un autre. 
Ce savoir diffère également en nature chez toute personne au gré des informations engrangées et, bien entendu, de celles oubliées.

En Saskatchewan, une connaissance de la géographie européenne s'avère utile pour associer les noms d'arrondissements scolaires que sont Aisne, Kemmel Hill, Lens, Metz, Mons, Poelcapelle, et Ypres à une culture d'expression wallonne ou française. Soulignons, au passage, qu'un nombre considérable de patronymes appartenant à des ressortissants de France portent en eux-mêmes un héritage qui a bien peu à voir avec la langue française. Ainsi, il faut être au fait d'une histoire qui n'est plus très récente pour être en mesure d'établir le lien qui unit étroitement l'hydronyme saskatchewannais Kleczkowski et l'influence de la France au Canada, tout comme il s'avère utile d'être familier de l'histoire des francophones de la Saskatchewan pour reconnaître et associer les toponymes Beynes, Oxarat ou Ponteix à la France, aux francophones et à leur histoire. Il faut être familier des dénominations autochtones et de leur évolution pour reconnaître dans le nom Illinois accordé à un ancien arrondissement scolaire, une adaptation phonétique française du désignatif Iliniwek.

Les limites d'un savoir peuvent être à l'origine d'une méprise, d'un fourvoiement. et amener à trop rapidement conclure à la présence d'erreurs de transcription. Une orthographe semblant s'éloigner de celle généralement admise peut parfois s'avérer une bonne transcription de la langue parlée. Gardipee Crossing $\left(52^{\circ} 52^{\prime} / 106^{\circ} 03^{\prime}\right)$ (pour Gariépy), qui désigne une ancienne traverse près de St. Louis, est un exemple qui évoque la prononciation de certains Métis francophones, habitants des lieux.

Les limites d'une culture personnelle, l'accès aux informations dans les méandres de sa propre mémoire, constituent une première entrave certes, mais elle n'est pas en cause lorsque l'accès aux toponymes eux-mêmes se fait avec difficulté.

Entraves géographiques - Lors même que tant de noms peuplent les cartes et foisonnent sur les continents, faible est le nombre de ceux d'entre eux qui connaissent un usage quotidien. Sont avant tout accessibles à l'usager de la toponymie, les noms 
familiers que l'on croise journellement ou qui font l'objet d'une médiatisation. Ils donnent corps à ce qui pourrait être qualifié de toponymie de proximité.

En Saskatchewan, l'écoumène occupe la moitié méridionale de la province. Les régions boréales, très peu peuplées, abritent de nombreux hydronymes. On y dénombre près de 100000 lacs. La majorité des toponymes d'origine et d'influence françaises se retrouvent au-delà de la frange septentrionale de l'écoumène, c'est-à-dire au nord du $54^{\mathrm{e}}$ parallèle, lequel traverse la province à mi-hauteur. Cet important référentiel toponymique identitaire demeure, en pratique, hors de portée d'ouïe et de vue. Ces noms ne peuvent participer aux échanges et à l'édification de l'habitus de l'endogroupe comme peuvent le faire les toponymes qui coiffent les lieux situés au cœur des centres de peuplement. Leur connaissance nécessite le recours à une médiation, celle de la carte. Léloignement des noms situés dans les régions boréales et l'indispensable recours à la carte réduit considérablement leur impact culturel au quotidien.

Il faut le souligner, la proximité demeure un état qui se mesure à différentes échelles. La distance géographique peut être un indice trompeur de familiarité. La médiatisation assure parfois plus favorablement l'usage de noms fortement éloignés que celui de noms peu distants, mais rarement évoqués. Qui connaît la majorité des noms de sa propre ville?

Entraves de nature mnésique - Plus de $60 \%$ des toponymes franco-saskatchewannais que nos recherches documentaires ont permis d'exhumer appartiennent au passé. Ils ne connaissent plus l'usage qui pourrait leur permettre d'être à nouveau évoqués au cours des échanges quotidiens qui rendent les mots familiers, représentatifs des manières de dire, de nommer et d'échanger. Permettre à une part appréciable du patrimoine toponymique répertorié de se retrouver à portée des oreilles et des mémoires nécessite un important travail de fouille et d'orpaillage. La remise en circuit des noms ayant déserté les cartes ou n’ayant jamais pu s’y inscrire pose des difficultés appréciables. Les plus insolubles, bien sûr, concernent les noms qui, ayant échappé à toute consi- 
gnation, sont relégués à jamais dans les oubliettes du temps. La fragilité des patrimoines oraux met à mal toute velléité de parvenir à des inventaires exhaustifs et à reconstituer au mieux les manières de nommer au fil des siècles. En situation de contact des langues, les traductions mettent à mal la mémoire des formes originelles lorsque le mot du groupe majoritaire parvient à évincer celui de la langue dans laquelle le nom avait pris forme.

\section{Tableau 1}

Toponymes maintenus et toponymes disparus en relation avec les périodes étudiées

\begin{tabular}{|l|c|c|c|c|c|c|}
\hline Période & Toponymes & $\%$ & Maintenus & $\%$ & Disparus & $\%$ \\
\hline $\begin{array}{l}\text { Ancien Régime } \\
(1699-1763)\end{array}$ & $\mathbf{2 0}$ & 1,0 & $\mathbf{0}$ & 0 & $\mathbf{2 0}$ & 100 \\
\hline $\begin{array}{l}\text { Traite des } \\
\text { fourrures } \\
(1764-1829)\end{array}$ & $\mathbf{1 8 0}$ & 9,2 & $\mathbf{1 3}$ & 7,2 & $\mathbf{1 6 7}$ & 92,8 \\
\hline $\begin{array}{l}\text { Métis } \\
(1830-1885)^{*}\end{array}$ & $\mathbf{2 0 0}$ & 10,2 & $\mathbf{2 0}$ & 10 & $\mathbf{1 8 0}$ & 90 \\
\hline $\begin{array}{l}\text { Missionnaires } \\
(1845-1915)\end{array}$ & $\mathbf{8 6}$ & 4,4 & $\mathbf{1}$ & 0,8 & $\mathbf{8 5}$ & 99,2 \\
\hline $\begin{array}{l}\text { Colonisation } \\
(1886-1929)^{*}\end{array}$ & 881 & 45,1 & $\mathbf{2 1 0}$ & 23,8 & $\mathbf{6 7 1}$ & 76,1 \\
\hline $\begin{array}{l}\text { Intervalle de crise } \\
(1930-1945)\end{array}$ & $\mathbf{1 4 1}$ & 7,2 & $\mathbf{9 9}$ & 70,2 & $\mathbf{4 2}$ & 29,8 \\
\hline $\begin{array}{l}\text { Bereskin } \\
(1946-1968)\end{array}$ & $\mathbf{3 7 9}$ & 19,4 & $\mathbf{3 4 6}$ & 91,3 & $\mathbf{3 3}$ & 8,7 \\
\hline $\begin{array}{l}\text { Époque } \\
\text { contemporaine } \\
(1969-2005)\end{array}$ & $\mathbf{6 5}$ & 3,3 & $\mathbf{4 9}$ & 75,3 & $\mathbf{1 6}$ & 24,7 \\
\hline \begin{tabular}{l} 
Total \\
\hline
\end{tabular} & $\mathbf{9 5 2}$ & 100 & $\mathbf{7 3 8}$ & 37,8 & $\mathbf{1 2 1 4}$ & 62,2 \\
\hline
\end{tabular}

* Les toponymes associés aux missions et dessertes de cette période sont reportés à l'entrée "Missionnaires".

Ainsi, un groupe peut voir sa toponymie oubliée tout comme il peut oublier les origines étrangères de noms qu'il croit appartenir en propre à sa langue et à sa culture. Une part appréciable des noms de lieux français recensés et qui remontent à l'époque 
de la traite des fourrures au $18^{e}$ siècle parait n'être en fait que des calques des amérindianymes en usage chez les peuples autochtones de l'époque.

Entraves liées à l'interprétation - Pour un emploi effectif du toponyme sur la carte comme dans les conversations, il n'est pas nécessaire que ce dernier affiche une transparence universellement attestée, ni qu'il affiche en toutes lettres les indications quant à son origine, à sa signification et aux particularités de son usage. La fonction première du nom, l'orientation, subordonne l'accès à de telles précisions. Elle conditionne sa présentation et la met en servitude. Ainsi, dépouillé, sans adjuvants contextuels explicites permettant la levée des ambiguïtés et sans indices qui permettent de le mettre en relation avec un patrimoine spécifique, quel qu'il soit, le toponyme oppose, au premier chef, une résistance à l'interprétation. Une désignation familière peut ne pas s'avérer d'interprétation malaisée si elle se charge de plus d'un sens. Baie Claire, cette appellation toute simple, peut se révéler d'interprétation ambigüe. Elle peut avoir été ainsi en raison de la limpidité de ses eaux ou encore pour rendre hommage à une personne dont le prénom est Claire. Isolé, " livré à lui-même ", le nom de lieu est exposé à des méprises de tous genres. Cette austérité de présentation, l'opacité qui en résulte et la vulnérabilité qu'elle engendre sont à l'origine même des toponymistes.

Entraves d'ordre épistémologique - Là où s'observent des frontières linguistiques imprécises, la différenciation des patrimoines toponymiques s'avère d'autant plus délicate que l'on est en présence de langues apparentées, caractérisées par une hétérogénéité lexicale et anthroponymique constitutive. Les emprunts linguistiques mutuels, voire à sens unique, se reflètent également dans la toponymie. Ils fragilisent les possibilités de procéder à une association assurée d'un nom à une langue ou à une autre. Le cas des termes génériques français "brûlé ", " butte ", " cache ", " coulée ", " chute ", " coteau ", " portage ", "prairie " et " rapides " sont au nombre des génériques de langue française empruntés par l'anglais nord-américain. Ils 
connaissent un usage répandu dans l'Ouest canadien ${ }^{15}$ et les appariements sont parfois étonnants; tel le vocable Prairie Butte $\left(52^{\circ} 14^{\prime} / 103^{\circ} 29^{\prime}\right)$ intégralement constitué de termes empruntés au français.

Les anthroponymes posent des défis étymologiques et épistémologiques majeurs. Tout comme les génériques qui viennent d'être évoqués, nombreux sont les noms et prénoms qui ont su traverser la barrière des langues ${ }^{16}$. Ils deviennent en quelque sorte apatrides. En Saskatchewan, Paul, Ernest, Jean, Louis et Thomas sont de ces noms désormais apatrides dont on a gratifié des lacs, des baies, des ruisseaux ou des rivières. La présence d'un générique dans la langue de la majorité entraîne des conséquences dont l'une est d'obscurcir l'identité des individus dont les noms constituent les spécifiques. Cet appariement fragilise le caractère emblématique de toponymes ainsi constitués puisqu'ils n'autorisent pas une association rapide et sûre du patronyme à la culture de la personne porteuse du nom. Il exerce, au contraire, une force centripète sur le nom favorisant ainsi l'association spontanée de la langue du spécifique à celle du générique.

L'exemple du toponyme saskatchewannais lac Kleczkowski illustre une tout autre embûche. Cet anthroponyme n'inspire d'aucune manière la présence d'un quelconque lien avec la culture française. Seule la présence du générique de langue française « lac » éveille des soupçons. Ce générique se démarque dans une région où presque tous les génériques sont de langue anglaise. Les soupçons trouvent leur justification dès que l'on est informé sur l'origine du nom de cette nappe d'eau qui fut ainsi nommée à l'occasion de la visite d'Alfred Kleczkowski, consul général de France, en septembre $1896^{17}$.

$\overline{15}$ Alan Rayburn, "English Geographical Names in Canada with Generic Terms of French Origin ", Canadian Geographer, vol. 7, n ${ }^{\circ}$ 2, 1970, p. 88-104. Voir aussi Pierre Daviault, "L'élément canadien-français de l'anglais d'Amérique ", Mémoires de la Société royale du Canada, vol. xlvi, troisième série, juin 1952, première section, Ottawa, 1952, p. 5-18.

16 Jean-Marc De Foville, Les 1000 prénoms pour vos enfants. Les 1000, Paris, Hachette, 1990, p. 182.

17 Romeo Bédard, History of Montmartre, Sask., 1893-1953, Regina, [s.n.], 
Les noms des localités Jasmin ( $\left.51^{\circ} 14^{\prime} / 103^{\circ} 39^{\prime}\right)$ et Fertile $\left(48^{\circ} 23^{\prime} / 101^{\circ} 27^{\prime}\right)$ pourraient même avoir été conçus pour remplir cette fonction. Ils s'y prêtent d'autant plus que l'absence de génériques pour les localités habitées autorise, avec de pareils noms, l'affichage d'une "neutralité d'apparence ".

Certains noms propres semblent ne pouvoir être facilement associés à la langue française et ne pouvoir trouver place sous le parapluie identitaire étudié. Tout est alors affaire de contexte. Le point ferroviaire Zola $\left(50^{\circ} 24^{\prime} / 105^{\circ} 39^{\prime}\right)$ en fournit un exemple. Pour justifier sa place au répertoire fransaskois, cet anthroponyme aux origines extra-hexagonales doit impérativement évoquer l'écrivain et sa contribution magistrale à la littérature française. De même, ce n'est qu'en référence à leur emprunt intra-hexagonal que les toponymes issus de patronymes d'origine bretonne Cam Lake ( $\left.54^{\circ} 05^{\prime} / 106^{\circ} 48^{\prime}\right)$, Guyader Lake (54 $\left.38^{\prime} / 102^{\circ} 54^{\prime}\right)$ et Lebret $\left(50^{\circ} 45^{\prime} / 103^{\circ} 42^{\prime}\right)$ y trouvent eux-mêmes leur place.

Les limites de l'individu, les frontières interlinguistiques indécises et le fonds commun interculturel ne circonscrivent pas l'ensemble des difficultés qui gênent la reconnaissance d'un héritage toponymique. D'autres obstacles se posent. Ceux que nous verrons à présent appartiennent au traitement soit volontaire, soit accidentel que l'on fait subir aux noms de lieux.

\subsection{Entraves d'ordre graphique}

L'orthographe des toponymes est sujette à modifications. Même si l'officialisation contribue à assurer une certaine pérennité formelle, il arrive qu'elle consacre des formes erronées parfois difficiles à corriger. Les altérations peuvent être accidentelles ou intentionnelles.

Altérations graphiques accidentelles - Les altérations graphiques, tout comme les dérives phonétiques dont elles sont parfois issues, entament l'intégrité référentielle du toponyme. Certaines dénominations sont particulièrement sujettes aux altérations et aux attractions paronymiques. Les " difformités " graphiques n'entrainent cependant pas toutes des conséquences

1953, p. 31-32. 
préjudiciables. L'oubli d'une lettre ou son redoublement, la substitution de voyelles ou d'une consonne ne représentent parfois que des dégradations bénignes à faibles incidences. La graphie de Belliveau Lake "Béliveau » (5330' / 109 16') embarrasse momentanément le chercheur, mais n'entrave pas de manière compromettante l'identification du référé si le patronyme est peu répandu et ses porteurs aisément identifiables. Bénignes chez les uns, ces légères métamorphoses peuvent entraîner une confusion conséquente chez d'autres noms. Il n'en tient qu'au changement d'une seule lettre, voire d'un signe diacritique, pour que les noms signifient autre chose. Cote Lake "Côté " (55 $27^{\prime}$ /

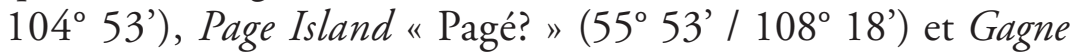
Lake "Gagné " (54 39' / $107^{\circ} 35^{\prime}$ ) en sont des illustrations.

Si la fonction du nom dicte en partie l'attitude à adopter à l'égard de formes sous lesquelles le toponyme se présente (le nom demeure performatif dans sa fonction de désignation), il n'en demeure pas moins qu'une graphie inattendue soulève des difficultés d'interprétation et d'association. Il arrive ainsi que des noms soient présentés sous des atours assimilables à la langue de la majorité. George Lake plutôt que "Georges Lake " (56 14' I $106^{\circ} 20^{\prime}$ ) en fournit un exemple. Dans de nombreux cas, l'absence ou la substitution d'un seul élément au jeu de lettres formant un patronyme suffit à rendre ce dernier totalement méconnaissable ou étranger. Cette entrave à l'identification avec un patronyme familier est apparente dans les noms de lieux Cedoux (49 $53^{\prime}$ I $103^{\circ} 52^{\prime}$ ), erreur de retranscription du toponyme Ledoux au North Dakota et Byers Ferry " au lieu de Boyer's Ferry " $\left(52^{\circ}\right.$ $\left.47^{\prime} / 106^{\circ} 06^{\prime}\right)$. L'ampleur du désastre causé par une modification graphique est variable. Il est rarement aisé d'en évaluer l'étendue de manière objective.

En d'autres circonstances encore, les apparentes dégradations graphiques peuvent apporter un concours inattendu à la connaissance ethnographique lorsqu'elles restituent le plus fidèlement possible une prononciation généralisée chez un groupe. Le toponyme révèle parfois sa "véritable " forme dans la langue parlée. Certaines graphies, lorsqu'elles ne sont pas le 
fruit d'une erreur de scribe, donnent des indications significatives sur la prononciation en usage à une époque reculée. Le toponyme Qu'Appelle River (50²8' / 101 29'), dérivé de " qui appelle » en raison de l'écho qui s'entend sur la rivière à la hauteur d'un certain lac, doit son existence et la pérennité de sa forme à une transcription par des locuteurs anglophones. Ils n'ont sans doute fait que copier la prononciation qui était celle des Métis habitant la région. Prenant le pas sur l'ancienne forme Qui Appelle ${ }^{18}$, le nom Qu'Appelle donné à la fameuse rivière, s'est finalement imposé. Cette transformation, si elle n'autorise pas une étymologie assurée mettant les Métis à la source même de la traduction de l'amérindianyme katepweositiy ${ }^{19}$, permet, tout au moins, de constater l'évolution d'un toponyme parmi les locuteurs métis francophones de cette région du centre-sud de la province.

Bien sûr, un toponyme à l'orthographe incongrue invite à l'enquête. Saswaus House ou Saswee House "François House " $\left(53^{\circ} 18^{\prime} / 104^{\circ} 02^{\prime}\right)$ nous informe sur la prononciation du prénom "François » chez les Cris et les Sauteux à l'époque de la traite des fourrures. Alors que, de prime abord, le nom semble entravé par une graphie déroutante, le toponyme, une fois interrogé, révèle plus qu'il n'y paraît.

En revanche, des noms peuvent sembler tout à fait en ordre et pourtant s'avérer des transcriptions fort éloignées du mot d'origine. Les toponymes Lake Miroir et Lac Meuron correspondent tous deux un lieu dont le véritable nom est Miron Lake (5506' I $\left.102^{\circ} 47^{\prime}\right)$.

En somme, les modifications sont dans la majorité des cas indésirables. À leur suite, l'on enregistre presque invariablement non pas un gain, mais un déficit. Le sens et l'évolution du nom souche ne se révèlent souvent qu'au prix de recherches laborieuses, si la chance n'est pas au rendez-vous. Roseray "Rose Roy "

18 Arthur Sylver Morton, "Five Fur Trade Posts on the Lower Qu'appelle River, 1787-1819 ", Comptes rendus de la Société Royale du Canada, vol. xxxv, troisième série, deuxième section, 1941, p. 82.

19 Irénée Tourigny, "Le père Joseph Hugonard, O.M.I. ", La Société canadienne d'histoire de l'Église cathotique / rapport 1948-1949, 1949, p. 23, note 2. 
$\left(50^{\circ} 28^{\prime} / 108^{\circ} 29^{\prime}\right)$, Napatak " pataque/patate, pomme de terre » $\left(55^{\circ} 02^{\prime} / 105^{\circ} 13^{\prime}\right)$, Tway "Touet " $\left(52^{\circ} 44^{\prime} / 105^{\circ} 26^{\prime}\right)$ ainsi que Saswee House $\left(53^{\circ} 18^{\prime} / 104^{\circ} 02^{\prime}\right)$ commenté ci-dessus l'illustrent bien.

Les exemples que nous venons de citer appartiennent pour la plupart au catalogue des noms officiels en usage. Nous terminerons ce thème par la présentation d'une collection de noms altérés. Sortis de l'usage, ils ont pu être recueillis sur des cartes anciennes, dans des journaux de bord anciens, des rapports de commis de traite, des lettres de missionnaires et de fonctionnaires de l'Administration. Nous offrons, entre guillemets, des indices d'interprétation.

Albertsville S.D. $N^{\circ} 3420$ "Albertville ", Rivière aux Biches "Wabish", Carriboeuf River "Caribou ", Gartou "Garonne? ", Ormaux S.D. N 2943 "Ormeaux ", Lac des Cauttes "Côtes? ", Capot River Fort "Cadotte?", Deleau "Delaleau?", Jubenville S.D. N 1155 "Jubinville», Lac de l'huile d'ours "Lac de l'île d'ours ", Mirond Lake "Miron ", Paulhaus Mine "Paulhus".

Altérations graphiques intentionnelles - Le détachement de l'autorité toponymique provinciale saskatchewannaise à l'égard de l'emploi des diacritiques français s'est appliqué de manière presque implacable, sans égard à la perte éventuelle d'informations étymologiques et historiques pertinentes ${ }^{20}$, ni au fait qu'un nombre considérable d'emprunts de l'anglais au français font en sorte que de nombreux toponymes intégralement français demeurent sémantiquement transparents et intelligibles à l'anglophone. Coulee Lake, Grand Coulee, Riviere des lacs, Legare Post en sont des exemples. Le sort réservé au signe diacritique, révélateur d'un état d'esprit chez les décideurs fut certes lourd de conséquences. Le sacrifice des signes diacritiques $^{21}$ cautionne l'adoption de formes inexactes et promeut, en

20 Jean Claude Bouvier, "Désignations onomastiques et identité culturelle ", dans Marianne Mulon, Françoise Dumas et Gérard Taverdet (dir.), Actes du colloque d'onomastique de la Société française d'onomastique, Paris, 1980, p. 22; Mark S. Monmonier, How to Lie with Maps, $2^{\mathrm{e}}$ éd., Chicago, University of Chicago Press, 1996 [1991], p. 110-111.

21 La Commission canadienne des noms géographiques (CCNG) décida, au début des années 1950, de permettre l'abandon des diacritiques pour les 
quelque sorte, une pratique qui n'est pas sans effet vexatoire au sein de la population des locuteurs de la langue à laquelle appartient le nom.

\subsection{Entraves d'ordre phonétique}

Le mot est un élément du langage relativement fragile. Comme le souligne le géographe Christian Morissonneau : "Chaque sujet parlant est facteur de changement dans la transmission orale $^{22}$. " Une élocution, trébuchante, incertaine, mal engagée peut être à l'origine de la transformation profonde d'un nom. Elle en atténue souvent la force évocatrice. Elle peut même en occulter l'étymologie qui en d'autres temps sauterait aux yeux. Partagées et transcrites, de telles dérives articulatoires peuvent connaître un usage relativement répandu, se retrouver sur la carte et faire l'objet d'une adoption officielle. Perran Lake $\left(53^{\circ} 28^{\prime} / 106^{\circ} 53^{\prime}\right)$ devrait normalement s'écrire "Perron

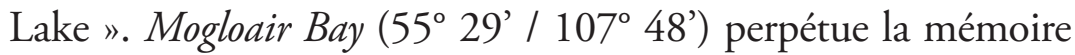
de Magloire Maurice, fondateur du poste de traite de la Compagnie de la Baie d'Hudson sur la rivière Gwillin. Peacard Lake $\left(49^{\circ} 43^{\prime} / 102^{\circ} 21^{\prime}\right)$ dans la région d'Arcola serait un toponyme dédicatoire perpétuant le souvenir d'Antoine Péchard, pionnier de l'endroit.

\subsection{Entraves d'ordre motivationnel}

Les altérations graphiques, tout comme les dérives phonétiques dont elles sont parfois issues, entament les propriétés référentielles des toponymes et peuvent aller jusqu'à modifier le sens originel des mots. Un toponyme saisi dans sa forme orale et transcrit par des locuteurs étrangers et inaptes à juger de la

toponymes en langue française dans les régions à prédominance de population anglophone. Saskatchewan Archives Board, Collection. R-190.4, Box 16, Canadian Board On Geographical Names. Lettre de G.M. Munroe, secrétaire du CBGN, à A. I. Bereskin, 19 octobre 1955.

Christian Morissonneau, "Noms de lieux et contact des langues : une approche de la choronymie du Québec ", Les noms de lieux et le contact des langues. Place Names and Language contact, Québec, Presses de l'Université Laval, 1972, p. 251. 
conformité d'un mot en regard des normes orthographiques dans la langue d'origine peut connaître des altérations qui occultent son véritable sens et son origine. Les resémantisations et les remotivations qu'elles relèvent, d'inductions endogènes ou exogènes ${ }^{23}$, figurent au nombre des difficultés qui entravent la saisie du patrimoine toponymique identitaire. Certains noms contiennent en eux-mêmes les conditions qui ont mené à leur resémantisation. Le toponyme Cypress Hills qui désigne un massif de collines (49 $\left.40^{\prime} / 109^{\circ} 30^{\prime}\right)$ à l'extrême sud-ouest de la Saskatchewan nous en fournit un exemple. Ce nom est une traduction de Montagne de Cyprès, appellation en usage chez les Métis francophones au $19^{\mathrm{e}}$ siècle. Or, à cette époque, le terme " cyprès " était utilisé chez les francophones pour désigner une essence particulière, le pin gris (pinus banksiana) qui, en anglais, se nomme familièrement jack pine. Conscient de la différence, les locuteurs anglophones de l'époque auraient probablement pu traduire Montagne de Cyprès par Jack Pine Hills.

Certains noms auxquels l'oreille d'une population est peu habituée sont parfois victimes d'incompréhension. Leur rendu articulatoire difficile peut faire subir à ces noms des adaptations compromettantes qui en obscurcissent le sens et la facture originelle. La désignation Saswee House évoquée précédemment en constitue un parfait exemple.

Ainsi la fracture de la mémoire ne peut être seule mise en cause dans la genèse des remotivations. La polysémie des mots, leur évolution, leurs évocations et la nature même du terrain conditionnent le rapport du nom au lieu et en influence l'interprétation.

$\overline{23}$ L'on entend par remotivation à induction endogène celle issue de conditions inhérentes au nom et pouvant mener à son éventuelle resémantisation. Les mots qui possèdent plusieurs sens ou susceptibles d'être confondus avec d'autres mots présentent cette caractéristique. Une remotivation à induction exogène s'observe le plus souvent lorsque les conditions à l'origine de la remotivation sont postérieures à l'attribution du nom dans sa forme originale. Carol Jean Léonard, "Typologie des modes de remotivation en toponymie/une illustration : la toponymie franco-saskatchewannaise ", ICOS 2005 Atti Del Xxii - Congresso Internazionale Di Scienze Onomastiche, Pise, Italie, 2008, p. 615-628. 
La toponymie d'une minorité linguistique semble plus sujette aux aléas des remotivations et des resémantisations du fait des difficultés que représente le passage d'une information motivationnelle ou sémantique d'une langue à une autre.

Il n'est pas aisé de rendre compte de la diversité des situations du fait même de la nature des remotivations, lesquelles ne peuvent être identifiées qu'après élucidation des transformations.

\section{Patrimoines enchevêtrés}

Le corpus toponymique minoritaire fransaskois constitue un matériel d'une saisie qui ne se révèle pas aisée. Sa justification repose sur des ressorts qui relèvent de phénomènes et de systèmes évolutifs, complexes, instables et "métissés". Si l'appropriation du patrimoine s'en trouve entravée, cela est en partie attribuable au fait que certains toponymes se présentent d'une manière que plus d'un groupe peut y voir "son héritage ». On peut voir en eux ce que l'on pourrait qualifier de " ponts interculturels " qui font porter le regard au-delà des murs qui délimitent les possessions identitaires nationales.

\subsection{Homéotoponymie}

À cet égard, l’on peut considérer les homéotoponymes comme des « aires » de partage et d'indifférenciation. Ainsi, l'appropriation du patrimoine toponymique fransaskois nécessite à la fois le recours à une gamme de savoirs et d'outils transdisciplinaires, tout comme elle requiert une certaine disposition pour faire la part des choses et ainsi mieux identifier la part de "soi ", la part de "l'autre " et ce qui constitue, en quelque sorte, une " copropriété » issue du vivre ensemble.

\subsection{Emprunts interlinguistiques}

Les termes génériques français (butte, cache, coteau, portage, etc.) évoqués précédemment fournissent un exemple d'un patrimoine toponymique communément partagé dont on ne peut cependant affirmer l'équité de traitement, en raison du délestage des diacritiques (brule, coulee) et même de lettres (rapids) qui 
affectent certains d'entre eux. S'ils ne sont pas issus d'un partage librement consenti, ils n'évoquent pas moins l'emprunt et l'adoption par le groupe majoritaire d'une manière de nommer jugée propre à devenir une façon nouvelle de dire qui constituera à son tour, au fil de son usage, un patrimoine commun nouveau.

\subsection{Néonymie hybride}

Lors de la période de la colonisation qui s'est étendue des années 1890 à 1930 environ, il est arrivé que des groupes d'origines diverses convergent vers de mêmes lieux, guidés par une communauté d'intérêts : proximité d'une voie ferrée, eaux abondantes, terres réputées fertiles ou même rareté des concessions encore vacantes. Ces fusions de convenance ont parfois trouvé leur écho dans la toponymie locale. Le recours à l'hybridation et à la segmentation a permis de créer une néonymie formée d'acronymes pour la composition desquels on emprunta soit des lettres, soit des syllabes. En voici quelques exemples: Alwinsal (composé des deux ou trois premières lettres des trois mots Alsace, Wintersall et Salzfurther), Duvco (assemblage créé à partir de Duval et Cory) Hamlin (de l'union d'Hamilton et Nolin), Rouworth S.D. (formé à partir de Rouleau et Shuttleworth), enfin, Kemoca Regional Park (conçu de la troncation de Kendal, Montmartre et Candiac).

L'hybridation n'est pas toujours perceptible. Elle se dissimule parfois sous des acronymes. Le nom Vawn, attribué à un petit village situé à une cinquantaine de kilomètres au nord-ouest de North Battleford, est un acronyme dont chacune des lettres est tirée des noms de pionniers de l'endroit, deux anglophones et deux francophones : Louis Vallier, Oscar Anderson, William Kruger et X. Nadon.

Les acronymes ne révèlent pas toujours leur véritable nature. Cela se produit lorsque l'agencement des lettres est tel qu'on en forme un mot d'usage commun. L'acronyme offre alors un couvert à son identification. Il se voit, pour ainsi dire, "soustrait » à la vue comme à l'oreille. Derrière chaque lettre formant le nom de l'ancien arrondissement scolaire Comfort S.D. $N^{\circ} 3316$ se cachait, semble-t-il, la première lettre du nom de 
colons francophones dont les enfants fréquentaient l'école de l'endroit ${ }^{24}$.

Ainsi, l'hybridité porte en elle le problème épineux du départage linguistique. En contexte de contact des langues, qui en est un de partage plus ou moins consenti, le départage du « nom en commun " apparaît vite litigieux. Si cette hybridité conduit à soulever le problème délicat de l'appropriation, elle s'opère au moyen d'une fusion de termes auxquels on attache un prix.

\section{Conclusion}

Si tant est que l'on puisse dissocier culture et langue ${ }^{25}$, cette dernière remplit une fonction référentielle majeure utile à qui souhaite identifier un patrimoine toponymique en contexte de contact interlinguistique. En raison de sa complexité, la toponymie des groupes linguistiques minoritaires exige, pour qu'on en saisisse le délinéament, un balisage au moyen de repères qui empruntent tant à la phénoménologie qu’à la lexicologie ou à l'étymologie. Le catalogage des toponymes selon leur appartenance linguistique requiert que l'on élucide les défis que posent l'anthroponymie, la toponymie d'origine dialectale, l'emprunt à diverses langues et l'hybridité linguistique. La saisie du corpus toponymique minoritaire fransaskois offre l'exemple de l'étendue des défis. Du fait même des caractéristiques essentielles, à la fois culturelles et linguistiques de son objet, toute tentative de circonscrire cette toponymie dans sa globalité et de la répertorier de manière exhaustive ne peut complètement se matérialiser. Son hétérogénéité constitutive, son métissage, son éloignement, son évanescence même rendent utopique d'en définir, dans leur totalité, les limites différenciatrices et exclusives. En pareil contexte d'acculturation et de transculturation, lorsque les prêts se mélangent aux emprunts et que les réinterprétations voisinent les combinaisons, la saisie du patrimoine dénominatif des lieux

24 Carol Jean Léonard, Mémoire des noms de lieux d'origine et d'influence françaises en Saskatchewan : répertoire toponymique, Québec, Les éditions GID, 2010, p. 156.

25 Jean Lafontant, "Langues, cultures et territoires, quels rapports? ", Cahiers franco-canadiens de l'Ouest, vol. 7, n' 2, 1995, p. 230. 
échappe à un enfermement culturel ${ }^{26}$ à qui prétend l'englober ${ }^{27}$. Parler "de l'ensemble des noms de lieux français " en Saskatchewan apparaît dès lors relever d'un syncrétisme et d'une fiction simplificatrice. De plus, toutes les conditions semblent s'être réunies au cours de l'histoire pour faire passer cette toponymie de la lumière à l'ombre : la disparition progressive d'une part importante de cette toponymie, sa nordicité, son éloignement des zones habitées, les emprunts, la traduction et la décapitation des signes diacritiques dont elle a fait l'objet y sont pour quelque chose.

Là où il y a contact de langues, là où les cultures s'adossent puis s'interpénètrent, les occasions de quiproquo référentiel sont fréquentes et ne peuvent souvent qu'être difficilement contournées. Du fait qu'elle ne rejoint pas aisément ceux qui peuvent s'en réclamer, la toponymie minoritaire fransaskoise connaît des conditions qui l'empêchent de figurer à l'avant-scène des "virtualités d'un substrat mythique, légendaire, artistique, où la communauté puise l'intuition de son identité ${ }^{28} \%$. Les Fransaskois sont davantage exposés à un paysage linguistique marqué par la langue de la majorité. La stabilité et la proximité de celui-ci font en sorte qu'il peut afficher avec efficacité un discours symbolique implicite traduisant le pouvoir de représentation et le statut des différentes communautés linguistiques en présence.

Par-delà les difficultés qu'éprouvent les Fransaskois à la saisie de leur patrimoine toponymique, la tentative de construction d'un répertoire offre non seulement et malgré tout une meilleure

26 Fernand Ouellet, " Pour éviter les pièges du relativisme culturel ", dans Françoise-Romaine Ouellette et Claude Bariteau (dir.), Entre tradition et universalisme, Québec, Institut québécois de recherche sur la culture, 1994, p. 154.

27 Jean-Jacques Simard, « La révolution pluraliste : une mutation du rapport de l'homme au monde ", dans Fernand Ouellet (dir.), Pluralisme et école : jalons pour une approche critique de la formation interculturelle des éducateurs, Québec, Institut québécois de recherche sur la culture, 1988, p. 41.

28 Michel Ottens, "La Wallonie dans les lettres françaises de Belgique ", Littératures en Wallonie, Louvain-la-Neuve, publications de l'Institut de littérature, 1981, p. 11. 
prise sur ce legs identitaire, mais, de plus, elle révèle qu'en dépit des efforts et des prétentions, nul groupe culturel ne peut prétendre détenir en propre l'ensemble des dénominations qu'il aura contribué à créer et à recréer. Miroir de l'identité de l'un, la nomenclature minoritaire est aussi miroir de l'autre dans la réaction qu'a le groupe majoritaire à l'égard d'une toponymie qui lui est étrangère, mais qu'il s'approprie et contribue parfois à pérenniser tout en lui faisant subir, dans certains cas, des altérations plus ou moins préjudiciables.

L'étude de la toponymie en tant que reflet identitaire, qu'il s'agisse ou non de celle de groupes linguistiques et culturels minoritaires, offre l'occasion de constater que les toponymes sont les témoins des dynamiques culturelles.

À l'exemple des cellules biologiques, les toponymies basées sur la langue et la culture possèdent un noyau solide dans lequel l'identité linguistique ne peut être mise en doute. Et, tout comme elles, les toponymies ont en leur pourtour ce qui ressemble étrangement à une membrane périphérique, lieu de nombreux échanges où se forge l'hétérogénéité de certains éléments lors de leur intégration. Dynamiques, hétérogènes, fruits d'une évolution complexe, ces toponymies favorisent à la fois l'identification tout en résistant à l'emmurement identitaire. Elles invitent à la réflexion sur les formes que prennent en toponymie les concrétisations de l'interculturalité.

\section{Bibliographie}

Bédard, Romeo, History of Montmartre, Sask., 1893-1953, Regina, [s.n.], 1953.

Bourhis, Richard Y., Howard Giles et Doreen Rosenthal, " Notes on the Construction of A "Subjective Vitality Questionnaire" For Ethnolinguistic Groups ", Journal of Multilingual and Multicultural Development, vol. 2, 1981, p. 145-155.

Bouvier, Jean Claude, "Désignations onomastiques et identité culturelle ", dans Marianne Mulon, Françoise Dumas et Gérard Taverdet (dir.), 
Actes du colloque d'onomastique de la Société française d'onomastique, Paris, 1980, p. 13-25.

Daviault, Pierre, "L'élément canadien-français de l'anglais d'Amérique ", Mémoires de la Société royale du Canada, vol. xlvi, troisième série, juin 1952, première section, Ottawa, 1952, p. 5-18.

De Foville, Jean-Marc, Les 1000 prénoms pour vos enfants. Les 1000, Paris, Hachette, 1990.

Dorion, Henri, "Les noms de lieux, repères indispensables dans l'espace et dans le temps ", Discours de conférencier prononcé à l'occasion du Géocongrès international, Québec, http://www.quebec2007.ca/pdf/ salle2000aetb/dinermercredi/h_dorion.pdf, (consulté le 20 juillet 2009).

Dorion, Henri, "La toponymie, complice involontaire de la politique ", dans Proceedings of the XIX $X^{\text {th }}$ International Congress of Onomastic Sciences, Aberdeen, 4 au 11 août 1996, Department of English, University of Aberdeen, 1998, p. 5-19.

Dorion, Henri et Christian Morrissonneau (dir.), Les noms de lieux et le contact des langues. Place Names and Language Contact, Québec, Presses de l'Université Laval, 1972.

Kadmon, Naftali "Toponymy and Geopolitics: The Political Use - and Misuse - of Geographical Names ", The Cartographic Journal, vol. 41, $\mathrm{n}^{\circ} 2,2004$, p. $85-87$.

Lafontant, Jean, "Langues, cultures et territoires, quels rapports?", Cahiers franco-canadiens de l'Ouest, vol. 7, no 2, 1995, p. 227-248.

Léonard, Carol Jean, Mémoire des noms de lieux d'origine et d'influence françaises en Saskatchewan; répertoire toponymique, Québec, Les éditions GID, 2010.

Léonard, Carol Jean, «Le patrimoine toponymique minoritaire d'origine et d'influence française à l'école : une voie de construction identitaire à emprunter ", Éducation francophone en milieu minoritaire, vol. 31, $\mathrm{n}^{\circ}$ 1, 2008, p. 15-32, http://www.reefmm.org/documents/EFMMleonard. pdf (consulté le 25 août 2009).

Léonard, Carol Jean, "Typologie des modes de remotivation en toponymie /une illustration: la toponymie franco-saskatchewannaise ", Icos 2005 - Atti Del Xxii Congresso Internazionale Di Scienze Onomastiche, Pise, Italie, 2008, p. 615-628.

Léonard, Carol Jean, "Une toponymie voilée : problématique des noms de lieux particulière à une minorité canadienne, la Fransaskoisie ", thèse de doctorat, Québec, Université Laval, 2006.

Monmonier, Mark S., How to Lie with Maps, $2^{\mathrm{e}}$ éd., Chicago, University 
of Chicago Press, 1996 [1991].

Morissonneau, Christian, "Noms de lieux et contact des langues : une approche de la choronymie du Québec ", Les noms de lieux et le contact des langues. Place Names and Language contact, Québec, Presses de l'Université Laval, 1972, p. 246-292.

Morton, Arthur Sylver, "Five Fur Trade Posts on the Lower Qu'appelle River, 1787-1819", Comptes rendus de la Société Royale du Canada, vol. xxxv, troisième série, deuxième section, 1941, p. 81-93.

Nicolaisen, Wilhelm Fritz Hermann, "Placenames and Politics ", Names, vol. 38, n 3, 1990, p. 193-207.

Ottens, Michel, "La Wallonie dans les lettres françaises de Belgique ", Littératures en Wallonie, Louvain-la-Neuve, publications de l'Institut de littérature, 1981, p. 7-22.

Ouellet, Fernand, "Pour éviter les pièges du relativisme culturel ", dans Françoise-Romaine Ouellette et Claude Bariteau (dir.), Entre tradition et universalisme, Québec, Institut québécois de recherche sur la culture, 1994, p. 151-170.

Rayburn, Alan, "English Geographical Names in Canada with Generic Terms of French Origin ", Canadian Geographer, vol. 7, n 2, 1970, p. 88-104.

Saskatchewan Archives Board, Collection. R-190.4, Box 16, Canadian Board on Geographical Names. Lettre de G.M. Munroe, secrétaire du CBGN, à A. I. Bereskin, 19 octobre 1955.

Simard, Jean-Jacques, "La révolution pluraliste : une mutation du rapport de l'homme au monde ", dans Fernand Ouellet (dir.), Pluralisme et école : jalons pour une approche critique de la formation interculturelle des éducateurs, Québec, Institut québécois de recherche sur la culture, 1988, p. 23-55.

Statistique Canada. Division des opérations du recensement 2002.

Tap, Pierre (dir.), Identité collective et changements sociaux, Toulouse, Privat, 1979.

Tourigny, Irénée, "Le père Joseph Hugonard, O.M.I. ", La Société canadienne d'histoire de l'Église cathotique I rapport 1948-1949, 1949, p. 23-38.

Vallières, Alain, «The Administrative Framework of a Central Toponymic Agency ", Canadian Documents for the United Nations Toponymy Courses, Ottawa, 1992.

Vuolteenaho, Jani, et Lawrence D. Berg, Towards Critical Toponymies Chapter 1, http://www.ashgatepublishing.com/pdf/SamplePages/ Critical_Toponymies_Ch1.pdf, (consulté le 15 juillet 2009). 